\title{
A Bayesian approach to predict the structural responses of FGM plates with uncertain parameters
}

Cite as: AIP Conference Proceedings 2204, 040013 (2020); https://doi.org/10.1063/1.5141586 Published Online: 10 January 2020

M. A. R. Loja, A. Carvalho, J. P. Neto, T. A. N. Silva, and M. Vinyas

\section{ARTICLES YOU MAY BE INTERESTED IN}

Metaheuristic optimal design of adaptive composite beams

AIP Conference Proceedings 2204, 040012 (2020); https://doi.org/10.1063/1.5141585

The study of microstructure and wear behaviour of titanium nitride reinforced aluminium composites

AIP Conference Proceedings 2204, 040014 (2020); https://doi.org/10.1063/1.5141587

Computational studies on laminar flow in micro-channel heat sinks for electronic cooling applications

AIP Conference Proceedings 2204, 040015 (2020); https://doi.org/10.1063/1.5141588

Challenge us.

What are your needs for periodic signal detection?

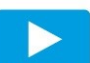

Watch

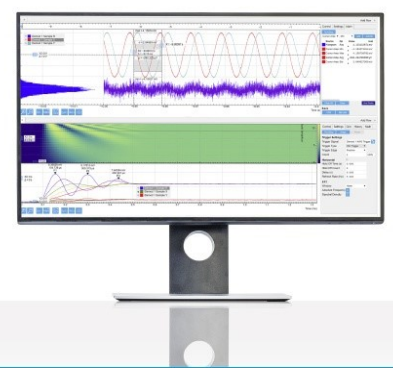

- Zurich - Instruments 


\title{
A Bayesian Approach to Predict the Structural Responses of FGM Plates with Uncertain Parameters
}

\author{
M.A.R. Loja ${ }^{1,2, \text { a) }}$, A. Carvalho ${ }^{1,3}$, J.P. Neto ${ }^{4}$, T.A.N. Silva ${ }^{1,2}$, M. Vinyas ${ }^{5}$ \\ ${ }^{1}$ CIMOSM - Centro de Investigação em Modelação e Optimização de Sistemas Multifuncionais, ISEL - Instituto \\ Superior de Engenharia de Lisboa, Av. Conselheiro Emídio Navarro, 1, 1959-007 Lisboa, Portugal. \\ ${ }^{2}$ UNIDEMI, Departamento de Engenharia Mecânica e Industrial, Faculdade de Ciências e Tecnologia, \\ Universidade NOVA de Lisboa, 2829-516 Caparica, Portugal. \\ ${ }^{3}$ CEMAPRE - Centro de Matemática Aplicada à Previsão e Decisão Económica, Universidade de Lisboa, Portugal \\ ${ }^{4}$ BioISI, Biosystems \& Integrative Sciences Institute, Faculdade de Ciências, Universidade de Lisboa, Portugal \\ ${ }^{5}$ Department of Mechanical Engineering, Nitte Meenakshi Institute of Technology, Bangalore, India - 560064. \\ ${ }^{a}$ Corresponding author: amelialoja@dem.isel.ipl.pt
}

\begin{abstract}
The increasing complexity associated to both structures and materials have been posing a continuous demand for numerical tools that allow predicting their behavior and simultaneously consider the influence of the uncertainty associated to multiple parameters. This work presents a Bayesian approach to characterize the influence of each material phase's properties and the volume fraction parameters in the behavior of biphasic functionally graded plates.
\end{abstract}

\section{INTRODUCTION}

Composites uncertainty assessment is a very contemporary research subject, and some works have become available in the literature. Nevertheless, functionally graded materials (FGM) have not deserved so far, as the authors' knowledge, an extensive attention from the research community. It is thus in this context and following previous works of the authors [1-3] wherein a classical perspective was considered, that one presents here a Bayesian approach in the characterization of the uncertainty influence in FGM plates' behavior.

\section{MATERIALS AND METHODS}

The FGM here considered, results from the mixture of two material phases' which are ruled by a power law [1]. To the tailor-ability characteristics, which they share with other types of composites, FGM can add other advantages and may be particularly attractive because of its potential to minimize stress concentrations arising from abrupt materials transitions and to its possible ability to be used in severe thermal environments.

To enable predicting the static and free vibration behavior of the mentioned power law FGM square plates, one has considered the use of in-house finite element codes, developed according the assumptions of the first order shear deformation theory [4,5]. The finite element used, is a nine nodes quadrilateral element, with five degrees of freedom per node, three translational midplane displacements associated to the three directions of the Cartesian coordinate system, and the remaining two degrees of freedom corresponding to rotations around the in-plane directions of the plates. These codes were already verified in $[1,3]$ concluding on its very good performance. 


\section{ON THE SIMULATION OF UNCERTAINTY}

Considering the modelling parameters as uncertain, one may assume that both mechanical and geometrical properties are randomly distributed to simulate the uncertainty on the FGM's properties. Among these parameters, one can find the exponent of the volume fraction distribution, which determines the inclusion rate through thickness of the reinforcement particles. For the present work, a FGM plate with aluminium matrix is used, being the ceramic inclusions of zirconia $\left(\mathrm{ZrO}_{2}\right)$. Additionally, the thickness, and all the mechanical properties of each constituent materials are also deemed to be uncertain. In this work, one has simulated the referred uncertainty on both mechanical and geometrical properties using a random multivariate normal distribution $\mathbf{X} \sim N(\boldsymbol{\mu}, \boldsymbol{\Sigma})$, with the vector $\boldsymbol{\mu}$ of mean values given in Tab. 1 and a diagonal covariance matrix $\boldsymbol{\Sigma}$ (ensuring the independence between modelling parameters) with standard deviations computed using a given coefficient of variation.

For sampling purposes, one has used a Latin Hypercube Sampling (LHS), often considered to perform simulations emulating physical systems. Note that this LHS implementation has the ability to ensure the independence between variables [6], besides the common advantage of a good coverage of the variables space.

TABLE 1. Mean values of the parameters used for simulation [7].

\begin{tabular}{cccccccc}
\hline Parameters & $E_{c}$ & $\begin{array}{c}v_{c} \\
\text { (ceramic - zirconia) }\end{array}$ & $\rho_{c}$ & $E_{m}$ & $v_{m}$ & $\rho_{m}$ & $p$ \\
(metallic - aluminium)
\end{tabular}

\section{REGRESSION MODEL}

A regression model aims at building a probabilistic model that relates a dependent variable $\mathrm{Y}$ to a predictor $\mathrm{X}$. As referred, the uncertainty on FGM plates may be due to several uncertain modelling parameters. Therefore, one proposes the use of a Bayesian approach to build a regression model. Hence, one uses a Bayesian multiple linear regression model to estimate the structural responses $\hat{y}_{i}$, this is achieved by the analysis of Bayesian hierarchical models using Markov Chain Monte Carlo (MCMC) simulation through JAGS [8]. In this work, estimate of the structural responses is given by

$$
\hat{y}_{i}=\beta_{0}+\sum_{j} \beta_{j} x_{i j}
$$

where the subscript $\mathrm{j}$ is the number of independent variables used to explain the dependent variable, $\beta_{0}$ is the intercept, which corresponds to the value predicted when the independent variables are zero, whereas $\beta_{j}$ are the regression coefficients, representing the influence of the parameter $x_{i j}$ on the predicted response $\hat{y}_{i}$.

As one is using a Bayesian regression model and to be more robust to outliers, a t-distribution is considered to model the observed structural responses $y_{i}$,

$$
\mathrm{y}_{\mathrm{i}} \sim \mathrm{t}\left(\mu_{\mathrm{i}}, \sigma, \tau\right)
$$

with

$$
\mu_{i}=\beta_{0}+\sum_{j} \beta_{j} x_{i j}
$$

where $\mu_{i}, \sigma$ and $\tau$ are parameters of the t-distribution. All the referred parameters have vague prior distributions, based on the available information, being 


$$
\begin{gathered}
\beta_{\mathrm{k}} \sim \mathrm{N}(0,2) \\
\sigma \sim \mathrm{U}\left(10^{-5}, 10\right) \\
\tau \sim 1+\operatorname{Exp}\left(\frac{1}{29}\right)
\end{gathered}
$$

The option to model $\tau$ with a shifted exponential distribution is to ensure that the degrees of freedom of the tdistribution are greater or equal to 1 . This Bayesian regression model is a hierarchical model function of a set of prior distributions and of the data, both modelling parameters and responses.

\section{RESULTS}

Considering the described in the previous section on the simulation of uncertainty, one has generated a sample of $\mathrm{n}=30$ observations of our multivariate random variable. As referred, one sampled geometrical and mechanical properties of the FGM's constituents, the moduli of elasticity, the coefficient of Poisson, the material density, the volume fraction exponent and the plate thickness. The mean values for these parameters are listed in Table 1. Note that the mean plate thickness is $\mathrm{h}=0.1 \mathrm{~m}$, and an aspect ratio $\mathrm{a} / \mathrm{h}=10$. All these parameters are sampled considering a multivariate normal distribution with a coefficient of variation of $7.5 \%$.

The choice for a sample size of 30 observations is related to the direct application with experimental data sets, for which the available data is often limited. Figure 1 shows a matrixplot of the sampled FGM properties. With the sampled modelling parameters, one has proceeded to the finite element analysis aiming at characterizing the maximum transverse displacement of the FGM plate (def in Fig. 1) and its fundamental frequency (f1 in Fig. 1).

In Figure 1, in the main diagonal of the matrixplot, it is shown the individual histograms of all variables, both modelling parameters and structural responses (the last two variables on the right of the matrixplot). Their scatterplots appear in the bellow the main diagonal, while in upper triangular part of the matrixplot one gives the

\begin{tabular}{|c|c|c|c|c|c|c|c|c|c|}
\hline$E_{c}$ & $r=0.0059$ & $r=-0.0018$ & $r=-0.0639$ & $r=-0.0249$ & $r=-0.0025$ & $r=0.0248$ & $r=0.0003$ & $r=0.2649$ & $r=0.2991$ \\
\hline mif & ${ }^{E_{\text {m }}}$ & $r=-0.0813$ & $r=0.0317$ & $r=0.0642$ & $r=-0.0467$ & $r=0.0061$ & $r=0.0051$ & $r=0.1484$ & $r=0.1575$ \\
\hline. & 80 & $\nu_{c}$ & $r=0.0107$ & $r=0.0009$ & $r=0.0266$ & $r=0.0496$ & $r=0.0611$ & $r=0.0791$ & $r=0.0725$ \\
\hline. & \% & \% & $\nu_{\mathrm{m}}$ & $r=0.0136$ & $r=-0.0290$ & $r=0.0442$ & $r=0.0085$ & $r=0.0151$ & $r=0.0274$ \\
\hline య & .2882808 & - & \% & $\rho_{\mathrm{c}}$ & $r=0.0465$ & $r=0.0392$ & $r=0.0104$ & $r=0.0235$ & $r=-0.2937$ \\
\hline \% & gீ: & 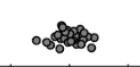 & 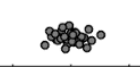 & 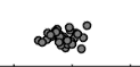 & $\rho_{\mathrm{m}}$ & $r=-0.0427$ & $r=-0.0084$ & $r=0.0145$ & $r=-0.1641$ \\
\hline 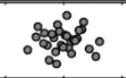 & अ.8 & . & so & 耑 & 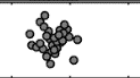 & p & $r=-0.0253$ & $r=-0.0539$ & $r=-0.0392$ \\
\hline 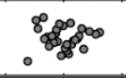 & 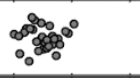 & \% & ) & 8: & \&\% & ○ంళి & h & $r=0.9344$ & $r=0.8787$ \\
\hline . & 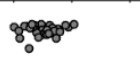 & .88 & $8088^{\circ}$ & 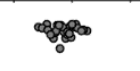 & 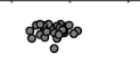 & . & & & $r=0.9201$ \\
\hline . & 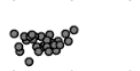 & . & s: & ఈำ & ) & 。 & 0 & $\infty$ & $f_{1}$ \\
\hline
\end{tabular}
correlation coefficients ( $\mathrm{r}$ ) of the data set. As expected, the individual histograms for the modelling parameters show a Gaussian behavior according to the values presented in Table 1 .

FIGURE 1. Characterization of the sampled modelling parameters and the resulting structural responses.

However, this is not that evident regarding the structural responses, as they are a nonlinear combination of the modelling parameters. From the correlation coefficients of the modelling parameters, it can be observed that these variables are uncorrelated $(r<0.085)$, as imposed to the LHS in the phase of simulation of uncertainty. Regarding 
the correlation coefficients of the structural responses, it may be concluded that the maximum transverse displacement and the fundamental frequency of the FGM plate are highly correlated $(r>0.9)$.

A preliminary analysis on the relation between modelling parameters and responses can be made by observing the correlation coefficients. Here, the dependency of the maximum transverse displacement and the fundamental frequency regarding the plate thickness is evident. This corroborates an expect result, as the plate stiffness is highly dependent on its thickness $(r>0.87)$, but also on the elastic moduli, especially the ceramic one (with a significant higher value). It is quite interesting to observe that the maximum transverse displacement is independent from the variation on the material density $(r<0.025)$, which is not the case of the fundamental frequency $(r>0.16)$. This is expected as the static responses are independent from mass parameters, while the dynamic ones are function of it. The reason why the correlation coefficients between the maximum transverse displacement and the material density parameters are different from zero is that they are all dependent from the volume fraction exponent $(p)$.

After this preliminary analysis of the data, the following discussion is concerned to the results of the Bayesian regression model. As referred, the regression coefficients in our Bayesian approach are themselves uncertain variables. Hence, regarding the MCMC method, one estimates posterior distributions for all the regression coefficients from the considered prior distributions and the available data, allowing to build our prediction model, which results are given in Figures 2 and 3.

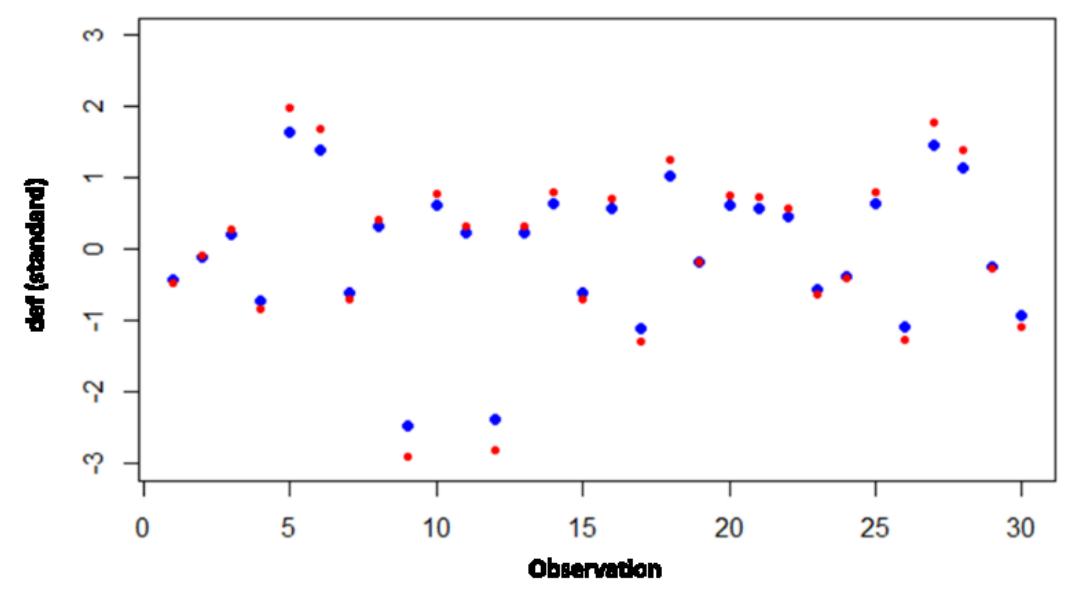

FIGURE 2. Standardized maximum transverse displacement (def): observed (blue) and predicted values (red).

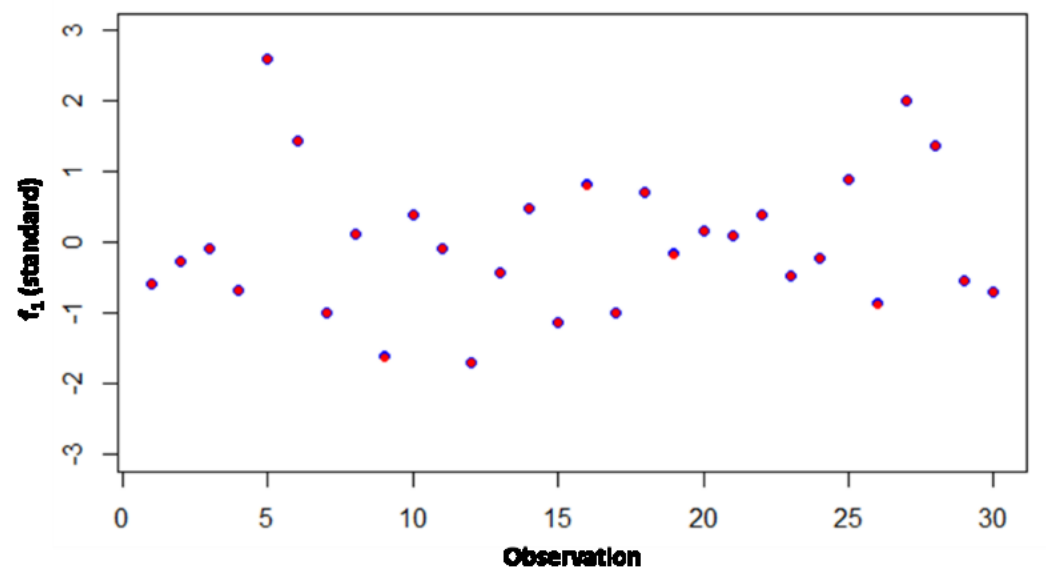

FIGURE 3. Standardized fundamental frequency (f1): observed (blue) and predicted values (red).

Note that each the regression coefficient is associated with a specific modelling parameter of the FGM plate and their posterior distributions are shown in Figure 4. 

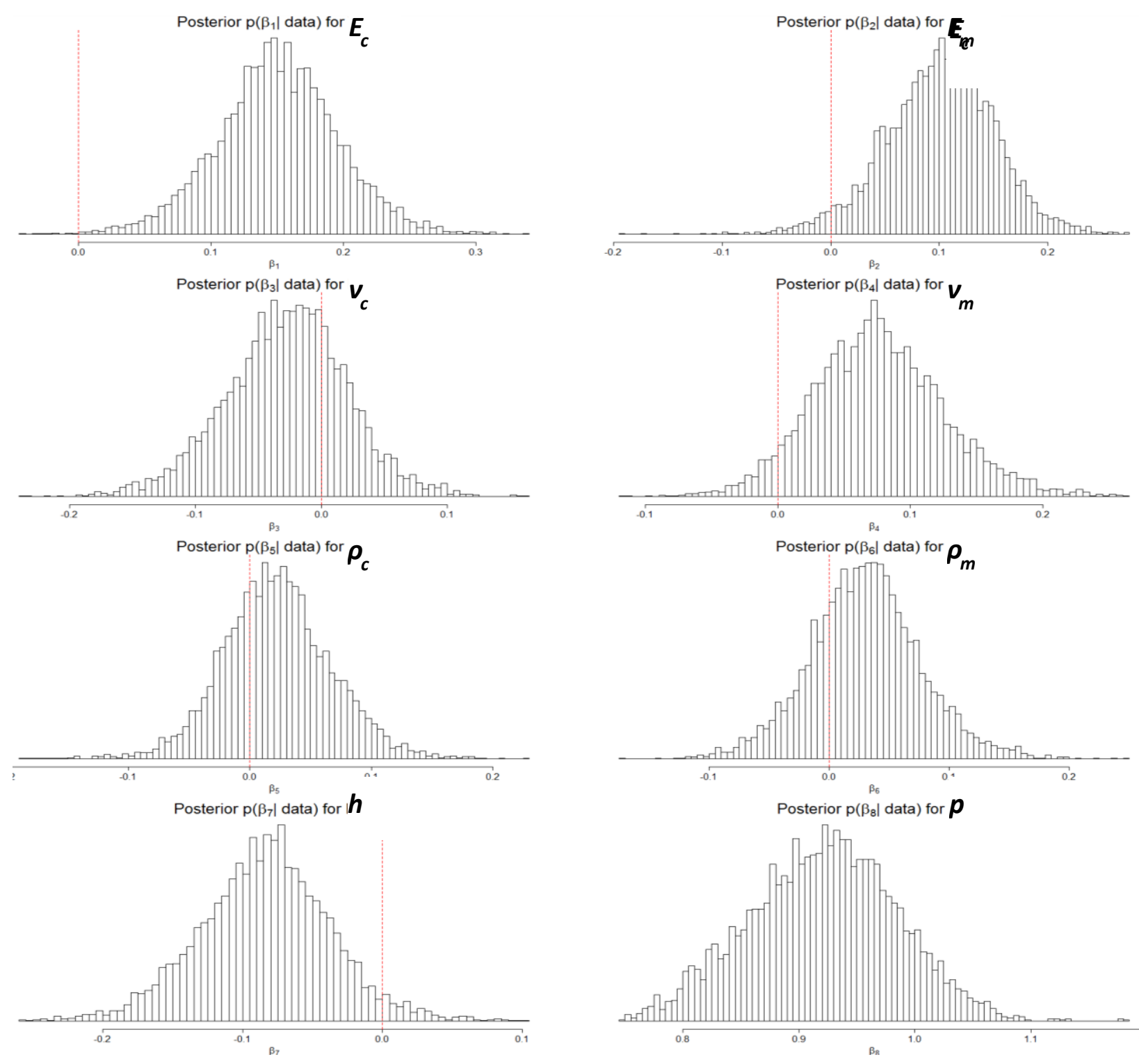

FIGURE 4. Posterior distributions for the regression coefficients associated with each FGM modelling parameter for the prediction of the maximum transverse displacement.

Figures 2 and 3 present a quite good estimate of the observed results for the considered structural responses. Note that using the proposed Bayesian approach one can estimate responses from a sample of modelling parameters in a less biased framework and compute the credibility intervals for that estimates.

For sake of simplicity, in this work, the posterior distribution for the regression coefficients are only given for the prediction of the maximum transverse displacement, as the ones for the prediction of the fundamental frequency are similar.

Superimposed to the posterior distributions of Figure 4, one has a vertical line at zero, allowing to observe the most influent modelling parameters on the description of the variability of each structural response. Note that a regression coefficient with a posterior centered around zero is statistically non-significant in the description of such variability. 


\section{CONCLUSIONS}

The prediction of structural responses by computationally efficient methods is of great interest for several fields of application. The ability to analyze and to make informed decisions from a virtual prototype may avoid different restrictions in the design phase of complex materials and products. FGM may provide the combination of interesting advantages of their constituents thus deserving a strong investment in research and development.

The present work presents a hierarchical Bayesian multiple linear regression model, to estimate the static and free vibrations behavior of squared FGM plates.

The trained Bayesian regression model allows for the prediction of structural responses in a less biased framework, when compared with classical regression models, where one must comply with several assumptions.

Additionally, from the analysis of the posterior distributions of the considered regression coefficients, one may conclude on the statistical significance of each modelling parameter on the variability of the structural responses, and all these in a design phase, where several modelling and optimization options are still available for implementation.

\section{ACKNOWLEDGEMENTS}

The authors wish to acknowledge the financial support given by FCT/MEC through Projects: UNIDEMI UID/EMS/00667/2019 and CEMAPRE UID/MULTI/00491/2019. The first author also wishes to thank the support of Project IPL/2019/MOCHVar/ISEL.

\section{REFERENCES}

1. Carvalho, A., Silva, T.A.N., Loja, M.A.R, Damásio, F.R.: Assessing the influence of material and geometrical uncertainty on the mechanical behavior of FGM plates. Mech. Adv. Mater. Struct. 24, 417-426 (2017).

2. Rosa, R.S.B., Loja, M.A.R., Carvalho, A.: Toward Variability Characterization and Statistic Models' Constitution for the Prediction of Exponentially Graded Plates' Static Response. J. Compos. Sci. 2, 59 (2018)

3. Carvalho, A., Silva, T.A.N., Loja, M.A.R.: Assessing static and dynamic responses variability due to parametric uncertainty on fibre reinforced composites. J. Compos. Sci. 2, 6 (2018).

4. Mindlin, R.D.: Influence of rotary inertia and shear on flexural motion of isotropic elastic plates. J. Appl. Mech, 18, 31-38 (1951).

5. Reissner, E.: The effect of transverse shear deformation on the bending of elastic plates. J. Appl. Mech. 13, 69-77 (1945).

6. Iman R.L., Conover, W.J.: A distribution-free approach to inducing rank correlation among input variables. Commun. Stat. B-Simul. 11, 311-334 (1982).

7. Bernardo, G.M.S., Damásio, F.R., Silva, T.A.N. and Loja, M.A.R: A study on the structural behaviour of FGM plates static and free vibrations analyses, Compos. Struct. 136, 124-138 (2016).

8. J. Kruschke, Doing Bayesian Data Analysis, Academic Press (2015). 